\title{
Vers un compte satellite des institutions sans but lucratif (ISBL) en France (I ${ }^{\mathrm{re}}$ partie) \\ Towards a satellite account of nonprofit institutions (NPIs) in France (part one)
}

\section{Edith Archambault et Philippe Kaminski}

Numéro 293, juillet 2004

De l'association à l'entrepreneuriat social

URI : https://id.erudit.org/iderudit/1022096ar

DOI : https://doi.org/10.7202/1022096ar

Aller au sommaire du numéro

Éditeur(s)

Institut de l'économie sociale (IES)

ISSN

1626-1682 (imprimé)

2261-2599 (numérique)

Découvrir la revue

Citer cet article

Archambault, E. \& Kaminski, P. (2004). Vers un compte satellite des institutions sans but lucratif (ISBL) en France ( $\mathrm{I}^{\mathrm{re}}$ partie). Revue internationale de l'économie sociale, (293), 10-23. https://doi.org/10.7202/1022096ar
Résumé de l'article

Que la comptabilité nationale permette d'évaluer le poids économique des activités d'économie sociale constitue un enjeu pour l'ensemble des organisations du secteur. Même si cet objectif reste encore hors d'atteinte, une avancée significative a été accomplie, puisque l'Union européenne incite les États-membres à mettre en oeuvre, dans le système des comptes nationaux, le " Manuel des institutions sans but lucratif " (ISBL) tel qu'adopté par l'ONU en 2002. Autoriser une comparaison à l'échelle internationale n'est pas le moindre mérite du compte satellite des ISBL, qui, pour les auteurs, ouvre la voie au compte satellite de l'économie sociale pour lequel milite et travaille l'Addes depuis plus de vingt ans. Avant de s'intéresser au « Manuel », l'article précise les concepts qui prévalent dans le champ de l'économie sociale et les différentes acceptions dans les pays. Actuellement, seules les institutions sans but lucratif au service des ménages (ISBLSM) sont lisibles dans les comptes nationaux. Comment passer au compte des ISBL ? Comment situer l'ISBL par rapport à l'association? Autant de questions qui sont explorées dans cette importante contribution, présentée au colloque de l'Addes le 15 décembre 2003, que nous publions en deux parties (la seconde partie paraîtra dans le numéro 294). 


\section{VERS UN COMPTE SATELLITE DES INSTITUTIONS SANS BUT LUCRATIF (ISBL) EN FRANCE}

(Ire partie)

(*) Présidente de l'Association pour le développement de la documentation en économie sociale (Addes). $\left.{ }^{* *}\right)$ Vice-président de l'Addes. par Edith Archambault ${ }^{(*)}$ et Philippe Kaminski ${ }^{(*)}$

Que la comptabilité nationale permette d'évaluer le poids économique des activités d'économie sociale constitue un enjeu pour l'ensemble des organisations du secteur. Même si cet objectif reste encore hors d'atteinte, une avancée significative a été accomplie, puisque l'Union européenne incite les Etats-membres à mettre en cuvre, dans le système des comptes nationaux, le "Manuel des institutions sans but lucratif " (ISBL) tel qu'adopté par l'ONU en 2002. Autoriser une comparaison à l'échelle internationale n'est pas le moindre mérite du compte satellite des ISBL, qui, pour les auteurs, ouvre la voie au compte satellite de l'économie sociale pour lequel milite et travaille l'Addes depuis plus de vingt ans. Avant de s'intéresser au "Manuel ", l'article précise les concepts qui prévalent dans le champ de l'économie sociale et les différentes acceptions dans les pays. Actuellement, seules les institutions sans but lucratif au service des ménages (ISBLSM) sont lisibles dans les comptes nationaux. Comment passer au compte des ISBL? Comment situer l'ISBL par rapport à l'association? Autant de questions qui sont explorées dans cette importante contribution, présentée au colloque de l'Addes le 15 décembre 2003, que nous publions en deux parties (la seconde partie paraîtra dans le numéro 294).

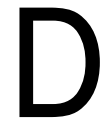

ès sa création, il y a maintenant plus de vingt ans, l'Addes s'était donné pour objectif de favoriser la construction en France d'un compte satellite de l'économie sociale. Pendant toute cette période, nous n'avons pas ménagé nos efforts pour convaincre nos interlocuteurs de l'intérêt et de la nécessité d'un tel instrument. A plusieurs reprises, au gré du renouvellement des responsables et après quelques avancées ayant fait malheureusement long feu, nous avons recommencé notre patient travail d'explication et de sensibilisation. Les circonstances ont évolué, notre argumentation s'est renouvelée, mais sur le fond nous n'avons jamais dévié de notre but. La chronique des rapports d'activité présentés à chacun de nos colloques témoigne de cette inlassable volonté d'aboutir un système permanent d'évaluation chiffrée des activités d'économie sociale, un tel système se devant, pour garantir son caractère objectif et scientifique, d'être articulé avec le corpus général de la comptabilité nationale. 


\section{Un compte satellite, enfin!}

Si notre pays joua jadis un rôle pionnier en matière de comptes satellites, s'il fut le premier à donner un cadre officiel à la notion d'économie sociale, il n'en est plus de même aujourd'hui, et c'est par le détour de la pression internationale que nous voyons enfin une fenêtre s'ouvrir. Là encore, l'Addes a joué un rôle moteur; Edith Archambault s'est trouvée dès l'origine correspondante pour la France du projet animé par l'université Johns Hopkins de Baltimore, projet dont la Recma s'est largement fait l'écho dans ses colonnes. Le réalisme et l'opiniâtreté de nos amis américains les ont amenés à faire adopter la synthèse de leurs travaux par l'ONU, sous forme d'un Manuel des institutions sans but lucratif dans le système des comptes nationaux, repris à son tour par l'Union européenne, qui souhaite le faire mettre en œuvre dans chacun des Etats-membres; ce qui a débloqué la situation en France, laquelle ne peut tout de même pas, aujourd'hui, se retrouver dernière de la classe.

Certes, le Manuel est assez nord-américain d'esprit. Certes, il ne traite pas d'économie sociale, mais de ce mystérieux agrégat que sont les institutions sans but lucratif (ISBL). Des esprits chagrins ne manqueront pas de le regretter. A coup sûr, il eût mieux valu aboutir en 1988, alors qu'un comité de pilotage officiel présidé par André Vanoli fonctionnait encore: il en serait sorti une construction intellectuelle bien adaptée à nos mentalités et à nos institutions, mais peut-être pas comparable internationalement. Cependant le mieux est l'ennemi du bien, et il faut savoir tirer le meilleur parti des occasions qui s'offrent à nous. Celle-ci vient à point nommé récompenser vingt ans d'efforts et d'attente: dès lors, nous ne bouderons pas notre plaisir.

Il ne s'agit encore, précisons-le, que d'une modeste et première étape: l'Insee s'est engagé pour une mission d'un an, couvrant l'année 2004, avec des objectifs limités. Il nous reviendra, le moment venu, le soin de définir les conditions de la poursuite, du développement et de la pérennité de cette initiative: raison de plus pour en assurer le bon déroulement.

Nous n'avons pas aujourd'hui de résultats à présenter (il faudra attendre début 2005); aussi, dans le présent article, bornerons-nous notre ambition à préciser le programme actuellement mis en œuvre et les notions que nous allons utiliser. Il était jusqu' ici question dans nos communications tantôt d'économie sociale, tantôt du monde associatif; désormais, nous avons affaire aux ISBL et à une partie de celles-ci, les ISBLSM. Nous vous proposons d'apprivoiser ensemble ces sigles insolites et les réalités qu'ils recouvrent.

\section{L'imbroglio des concepts: essai de clarification préalable à tout effort de mesure}

Définir l'économie sociale n'est pas simple parce que ce terme a deux acceptions: l'une, plus académique, correspond à un champ disciplinaire, alors 
que l'autre, plus institutionnelle, recouvre un ensemble d'organisations, coopératives, mutuelles, associations et fondations, qui se situent, pour faire court, entre l'Etat et le marché. C'est évidemment la deuxième acception qui nous concerne ici, mais dans la mesure où les deux termes ont une origine historique commune dans l'associationnisme ouvrier du XIX siècle, un détour vers la première n'est pas inutile.

\section{L'économie sociale comme champ disciplinaire}

Au sens académique, l'économie sociale tire ses origines de la distinction que fait Léon Walras dans ses trois œuvres fondamentales entre économie pure, économie appliquée et économie sociale. Alors que les deux premiers ouvrages traitent de la recherche de l'équilibre optimum, le troisième, qui reflète les convictions socialistes de Walras, s'intéresse à la justice sociale, par opposition à l'efficacité économique. Pour Walras, la justice sociale passe par une meilleure attribution des droits de propriété et par une disparition de la rente foncière, revenu sans cause lié à la détention d'une ressource rare, la terre.

On peut rapprocher de Walras un de ses contemporains, Charles Gide, professeur d'économie lui aussi, qui entretint avec lui une correspondance importante. Charles Gide rédigea un rapport sur le palais de l'Economie sociale de l'Exposition universelle de 1900. Ce rapport repose sur une conception de l'économie sociale proche de celle de Walras, mais moins théorique. Pour Charles Gide, les institutions de progrès social décrites et illustrées dans le pavillon de l'Exposition universelle se répartissent, selon ses propres termes, en "quatre têtes de chapitres":

" $i$. Celles qui tendent à procurer de meilleures conditions dans le régime de travail.

ii. Celles qui tendent à procurer le confort sous toutes ses formes.

iii. Celles qui tendent à assurer la sécurité de l'avenir contre tous les risques sociaux.

iv. Celles qui tendent à sauvegarder l'indépendance économique [...].

A ce grand ceuvre social, trois facteurs ont collaboré:

1. L'association libre [...], car si elle est obligatoire, elle devient institution d'Etat;

2. Le patronage, et non le "patronat", car ce mode d'activité sociale comprend non seulement les actes des patrons proprement dits, mais de tous ceux qui par suite d'une supériorité sociale quelconque se trouvent en situation d'aider les autres;

3. Les pouvoirs publics, parce que ce mode d'activité sociale comprend non seulement l'action de l'Etat, mais celle des municipalités, des départements, de l'Assistance publique."

Ce texte montre clairement combien les deux sens du terme « économie sociale " ont été initialement imbriqués.

Plus généralement, on peut définir l'économie sociale comme un projet collectif datant de la fin du XIX siècle qui vise à combler le fossé existant entre l'affirmation d'une citoyenneté politique généralisée et les conditions 
d'existence qui rendent l'exercice de cette citoyenneté impossible pour de larges parties de la population. En adoptant la terminologie contemporaine d'Amartya Sen, on peut dire que l'économie sociale vise à transformer les libertés formelles en libertés réelles.

C'est en référence à cette conception que s'est progressivement identifiée l'économie sociale comme spécialité disciplinaire académique: enseignement et recherche en ce domaine combinent économie du travail et protection sociale, étudient les liens entre éducation, formation et emploi, avec une attention particulière portée aux inégalités sous toutes leurs formes et à la réduction de ces inégalités par les politiques sociales. Sur le plan statistique, ce champ est largement couvert par les Données sociales, qui se sont imposées comme l'une des toutes premières publications de référence de l'Insee.

\section{L'économie sociale comme champ institutionnel}

Alors que l'économie sociale institutionnelle relève également de ce projet collectif né il y a plus d'un siècle, c’est à la fin de l'année 1981 que le concept d'économie sociale entre, par un décret créant la Délégation interministérielle à l'économie sociale (DIES), dans le droit français. L'économie sociale désigne les coopératives, les mutuelles et les associations gestionnaires, c'est-à-dire celles qui ont des activités de production. C'est ce champ que Vienney et Weber tenteront de cerner afin de délimiter les frontières d'un futur compte satellite de l'économie sociale, lors du premier colloque de l'Addes, en 1983.

Quelques années plus tôt, de grandes institutions de ce secteur s'étaient rapprochées pour donner naissance au Comité national de liaison des activités mutualistes, coopératives et associatives (Cnlamca), qui publia en 1980 une "Charte de l'économie sociale ", résumé des principes communs aux organisations regroupées. L'économie sociale, terme qui fait référence explicite à Charles Gide et à l'Exposition universelle de 1900, n'est donc pas une création du gouvernement socialiste, même si celui-ci a encouragé le regroupement de ce type d'organisations et créé une tutelle politique qui prendra des formes diverses au fil des gouvernements successifs.

Léconomie sociale n'est pas non plus une spécificité française. Partout dans le monde, au Nord comme au Sud, à l'Est comme à l'Ouest, on trouve des formes d'associations populaires qui répondent à des besoins individuels ou collectifs. Partout dans le monde, les entreprises de l'économie sociale se retrouvent en force dans les mêmes secteurs d'activité: agriculture, crédit, assurance, prévention et protection sociale, éducation, santé, services sociaux, culture et loisirs, environnement, défense des droits individuels et collectifs, développement local et coopération internationale.

La visibilité de cette réalité commune est cependant obscurcie par la terminologie, extrêmement variable d'un pays à l'autre, et de surcroît évolutive en fonction des modes. Nous allons donc essayer de recenser les principaux termes en vigueur pour désigner la réalité de l'économie sociale ici et ailleurs. 


\section{L'imbroglio terminologique}

Les termes de l'encadré suivant désignent aussi bien l'ensemble du secteur que les organisations qui le composent. Ces termes ne font pas référence à un statut juridique, mais plutôt à une pratique sociale. L'existence même d'un secteur est elle-même parfois contestée. Dans cet encadré, on a tenté de regrouper de manière logique les termes qui, dans les divers pays du monde, désignent une même réalité institutionnelle, en regroupant les concepts qui, en français et en anglais, insistent sur une des caractéristiques des organisations concernées.

On s'est limité aux termes français et anglais; pour plus de diversité linguistique, on peut consulter Salamon et Anheier (1997).

Un premier groupe isole secteur et organisations charitables; le concept de charities est utilisé surtout au Royaume-Uni et dans les pays de l'ex-Commonwealth, où une commission accorde le statut de charity aux organisations œuvrant dans l'intérêt général. Aux Etats-Unis, avec le même sens, prévaut le terme philanthropy, moins connoté avec les origines médiévales européennes, et au Japon celui de koeki hodgin avec la même acception. Tous ces termes insistent sur l'importance du don charitable,

\section{L'imbroglio terminologique}

(Les termes désignent à la fois les organisations et le secteur dans son ensemble.)

- charitable

- indépendant
- Royaume-Uni: charities / charitable sector

- Etats-Unis: philanthropic organizations / philanthropy

- Japon: koeki hodgin

\section{- bénévole}

- voluntary sector: Royaume-Uni, Irlande, pays scandinaves

- tiers secteur / third sector

- (récent) troisième système / third system (UE)

- Etats-Unis: independent sector

- organisations non gouvernementales / NGOs

- (récent) société civile / civil society

- exonéré fiscalement

- Etats-Unis: tax-exempt sector

- sans but lucratif / non lucratif - nonprofit/ not-for-profit sector

- système de comptabilité nationale SCN/93, SEC/95

- sociale / solidaire
- économie sociale / social economy:

France, Belgique, Europe du Sud

- Union européenne

- (récent) économie solidaire, économie populaire:

UE, Brésil et Amérique latine 
(1) Aussi l'association internationale regroupant les chercheurs et les professionnels travaillant sur ce domaine a-t-elle choisi de s'intituler International Society for third sector research (ISTR). Ce terme est construit sur le modèle de tiers état (ni aristocratie ni clergé) ou tiers monde (ni capitaliste ni communiste). Cette association internationale, qui initialement avait adopté une conception étroite proche du sens anglo-saxon, couvre actuellement le champ plus large de l'économie sociale.
(2) Le Comité économique et social européen, créé en 1957 par le traité de Rome, est à l'image du CES français. Il comporte trois groupes: le groupe des employeurs, le groupe des travailleurs lou " partenaires sociaux ") et le groupe des activités diverses, "qui rassemble des représentants des PME, des agriculteurs, des professions libérales, des consommateurs et des organisations de protection de l'environnement, ainsi que des acteurs issus du monde associatif, des sociétés mutuelles et des sociétés coopératives ". évidemment spécifique à ce secteur. Cependant, tous les travaux statistiques montrent que les dons constituent rarement l'essentiel des ressources des organisations concernées, sauf si l'on y inclut la valeur monétaire du don de temps, le bénévolat.

Le qualificatif bénévole / voluntary est typiquement britannique; il est utilisé au Royaume-Uni, en Irlande, dans les ex-dominions britanniques et, plus récemment, dans les pays scandinaves, champions du bénévolat. $\mathrm{Ce}$ terme insiste sur l'apport essentiel du travail bénévole. Cependant, une grande partie des charities, celles qui ont le poids économique le plus lourd, emploient majoritairement et parfois exclusivement des salariés et seules les organisations de petite taille ne fonctionnent que grâce au bénévolat. Un deuxième bloc regroupe des termes qui caractérisent le secteur comme troisième force entre Etat et marché (tiers secteur, employé dans de très nombreux pays ${ }^{(1)}$, ou troisième système, terme actuellement en vogue dans les instances européennes, qui trouve son origine en Italie). Aux Etats-Unis, ce secteur est vu comme opposé au secteur public (independent sector y désigne l'organisation fédérative la plus importante de ce pays et, par extension, le tiers secteur américain). Le terme d'organisation non gouvernementale $(\mathrm{ONG})$ / non-governmental organization $(N G O)$ relève de la même logique; cependant, l'usage de ce terme est souvent restreint aux organisations intervenant dans le domaine international ou pour des causes internationales (de l'aide au développement à la résolution des conflits en passant par l'aide d'urgence, les organisations "sans frontières " et le commerce équitable). Pour cette même raison, c'est le terme qui est le plus utilisé dans les pays en développement.

On pourrait aussi rattacher à ce courant le concept assez vague, mais très à la mode en ce moment, de société civile, issu de la pensée d'Habermas. Le terme anglais de civil society est plus riche, civilétant aussi synonyme de civique en français (par exemple dans civil rights). Civil est alors opposé à public ou à étatique et non à militaire comme au sens étymologique. Selon les auteurs, la société civile inclut les associations et les syndicats, qui exercent une démocratie participative par opposition à la démocratie élective des représentants élus de la nation, ou s'élargit aux entreprises à but lucratif et, dans ce cas, le terme devient bien flou. C'est dans cette acception large que l'article 257 du traité de Nice décrit le Comité économique et social européen comme une institution dont les membres représentent les différentes composantes de la société civile organisée ${ }^{(2)}$.

Aux Etats-Unis, on rencontre fréquemment le qualificatif « exonéré fiscalement " (tax-exempt) pour désigner secteur ou organisations. C'est en effet le fisc qui y détermine quelles organisations ont droit à une exemption fiscale partielle ou totale, déterminant ainsi les contours du secteur. Dans la plupart des pays, les organisations sans but lucratif sont exonérées d'un certain nombre d'impôts (impôt sur les sociétés, TVA...). Mais cette caractéristique est évidemment très variable et le problème de fond est de savoir sur quels critères se fonde l'administration fiscale pour admettre l'exonération. 
(3) Qui devait initialement s'appeler secrétariat d'Etat à l'Economie sociale et solidaire. La petite histoire raconte que Guy Hascouet pressenti pour occuper ce poste, avait dû choisir entre économie sociale et économie solidaire le qualificatif qui lui plaisait le plus, Lionel Jospin trouvant le titre initial trop long.
Le terme de secteur (ou d'organisation) non lucratif (nonprofit) ou sans but lucratif (not-for-profit) est certainement le plus couramment admis. Il exprime que les organisations appartenant à ce secteur n'existent pas principalement pour dégager des profits à l'intention de leurs propriétaires, leurs membres ou leurs gestionnaires. Cette caractéristique est évidemment partagée avec l'ensemble du secteur public. L'adoption généralisée de ce terme tient sans doute au fait que c'est celui qui est retenu par le système international de comptabilité nationale SCN 93 / SEC 95. Dans les comptes nationaux, les institutions sans but lucratif au service des ménages (ISBLSM) constituent un secteur institutionnel, mais les ISBLSM ne regroupent qu'une faible partie des institutions sans but lucratif (ISBL)

Enfin, le concept d'économie sociale (ou d'organisations de l'économie sociale) s'est développé principalement en France, en Belgique et dans l'Europe du Sud avant d'être adopté par la Commission européenne. Il inclut des entreprises comme les mutuelles d'assurance ou de prévoyance, les coopératives de crédit ou de consommateurs ou encore les caisses d'épargne, qui, dans les pays anglo-saxons et dans une grande partie du monde, sont considérées comme des entreprises ordinaires. Si le concept d'économie sociale a été lancé en Europe par Jacques Delors, puis repris par Jacques Santer, il a été initialement mal accepté dans l'Europe du Nord et continentale, en particulier en Allemagne, où le terme d'économie sociale de marché (Sozialmarktwirtschaft) désigne une tout autre réalité, celle des entreprises capitalistes cogérées par le patronat et les syndicats.

Aussi la refonte de la Commission européenne par Romano Prodi a-t-elle fait disparaitre l'ancienne DG 23 (petites et moyennes entreprises et économie sociale), désormais éclatée entre deux directions générales. Dans la DG « Petites et Moyennes Entreprises » se retrouvent coopératives et mutuelles, même si elles sont de grande taille, alors que la DG " Affaires sociales » regroupe associations et fondations, même quand leur objet est totalement étranger au domaine social. Dans les textes européens récents, le terme " économie sociale " est souvent remplacé par le sigle Cmaf, pour " coopératives, mutuelles, associations et fondations ", ou par " troisième secteur » ou " troisième système ».

Ce renoncement n'est sans doute pas irréversible et il semble que l'on assiste actuellement à un retour du terme d'économie sociale dans les textes européens. Le terme d'économie sociale est de plus en plus utilisé par les pays en développement, où ce secteur est en forte expansion, et aussi par les pays d'Europe du Nord auparavant récalcitrants. D'autre part, le sigle très plat de Cmaf n'est qu'une définition en extension de l'économie sociale, qui fait bon marché du projet collectif inhérent à ce terme.

Récemment, le terme d'économie solidaire a été consacré par l'existence d'un éphémère secrétariat d'Etat à l'Economie solidaire ${ }^{(3)}$. Il a été lancé en France par les travaux de Bernard Eme et Jean-Louis Laville, qui ont tendance à opposer l'économie solidaire émergente (services de proximité, entreprises d'insertion ou de réinsertion, commerce équitable, systèmes d'échanges locaux, microcrédit) à l'économie sociale installée, plus ancienne 
et plus insérée dans le marché. Cette opposition n'a pas été retenue dans le champ de compétence du secrétariat d'Etat, qui couvrait l'économie sociale dans son ensemble.

Léquivalent américain de l'économie solidaire serait alors l'économie communautaire ou community development (Canada, Etats-Unis), tandis qu'en Amérique Latine le terme d'économie solidaire tend à se substituer au terme plus ancien d'économie populaire, notamment lors des forums sociaux mondiaux de Porto Alegre et de Bombay.

\section{L'économie sociale définie par ses principes}

L'objet de l'Addes reste bien l'économie sociale, en dépit de ces fluctuations terminologiques qui ne facilitent pas la compréhension de l'originalité des organisations qui la composent par le grand public. Nous définirons l'économie sociale par les principes qui la régissent, en actualisant la charte de l'économie sociale de 1980 par des textes plus récents, ainsi qu'avec quelques caractéristiques communément admises.

- L'adhésion volontaire des membres ou des sociétaires est un principe fondamental des organisations de l'économie sociale. C'est le principe historique de la " porte ouverte ", qui signifie libre entrée et libre sortie, ce qui élimine du champ de l'économie sociale les sectes, les caisses de sécurité sociale et les organisations paritaires (Assedic, Agirc, Arrco...).

- La solidarité entre les membres est un principe historique, ancré dans le mouvement ouvrier du XIX siècle et inspiré par la philosophie solidariste qui lui est contemporaine (Bourgeois, Leroux, Durkheim). C'est évidemment le principe de base de la mutualité. La solidarité peut se traduire pratiquement par une participation des membres proportionnelle à leur revenu et non au service qui leur est rendu. La solidarité peut parfois être opposée à la charité ou à la philanthropie, "où la main qui reçoit est toujours en dessous de la main qui donne", comme le dit un proverbe africain.

- La gestion démocratique des organisations de l'économie sociale s'exprime par le principe historique " un homme égale une voix ", rendu plus politiquement correct par " une personne égale une voix ». Ce principe traduit une opposition fondamentale au mode de décision dans les sociétés anonymes capitalistes, régies par le principe " une action égale une voix".

- L'autonomie de gestion signifie que les organisations de l'économie sociale ont leurs propres organes de gouvernance et qu'elles ne sont pas de simples pseudopodes d'une entreprise ou d'une administration. En particulier l'indépendance vis-à-vis des pouvoirs publics signifie que les organisations de l'économie sociale sont distinctes de l'Etat et des collectivités territoriales; elles peuvent recevoir un financement public important, voire exclusif, mais ce ne sont pas les représentants des pouvoirs publics qui en contrôlent les instances dirigeantes.

- Le principe de non-lucrativité ou de lucrativité limitée n’interdit pas la réalisation d'un profit (excédent, surplus), mais ce profit ne doit pas être distribué aux membres ou aux dirigeants (non-lucrativité stricte) ou il peut être 


\section{Principes de l'économie sociale}

- Liberté d'adhésion; principe de la porte ouverte.

- Solidarité entre les membres.

- Gestion démocratique selon le principe « un homme, une voix ».

- Autonomie de gestion et indépendance à l'égard des pouvoirs publics.

- Non-lucrativité ou lucrativité limitée. Le but de l'organisation n'est pas la distribution du profit, mais l'épanouissement des personnes et la réalisation de son objet social.

- A-capitalisme; le capital et les réserves sont impartageables.

distribué aux sociétaires de manière limitée, sous forme d'intérêt ou de ristournes par exemple (lucrativité limitée). Schématiquement, la nonlucrativité stricte concerne les associations et les fondations, alors que la lucrativité limitée concerne les coopératives et les mutuelles. Quelle que soit sa forme, ce principe signifie que la distribution du profit n'est pas le but de l'organisation, celui-ci étant d'abord l'épanouissement des membres et la réalisation d'un objet social qui combine, avec des pondérations différentes selon les organisations, les intérêts des membres et l'intérêt général.

- La-capitalisme exprime le fait que le patrimoine détenu par une organisation de l'économie sociale est un patrimoine collectif. En cas de dissolution de l'organisation, le capital ou les réserves accumulées ne peuvent être partagés entre les membres ou les sociétaires. Ce patrimoine ne peut quêtre dévolu à une organisation similaire.

Face à cette multiplicité de concepts, qui pour la plupart ne correspondent pas aux critères objectifs pris usuellement en compte par les opérations statistiques, le devoir du scientifique n'est pas de les hiérarchiser ou de choisir ceux qui sont les plus pertinents, mais de s'efforcer de les éclairer tous par les moyens les plus appropriés, afin que l'utilisateur puisse toujours trouver de quoi décrire la " poupée russe " qui lui convient le mieux. Destiné avant tout à permettre des comparaisons internationales, le Manuel de l'ONU se devait de tracer des limites précises entre ce qui fait partie du champ et ce qui lui est extérieur. Mais cette contrainte de bon sens ne doit pas être comprise comme une mutilation: c'est au contraire une condition de cohérence et de clarté. La mise en œuvre pratique d'une règle impose que chacun y soit soumis; dès lors, caractériser les unités qui font partie d'un champ revient ipso facto à caractériser aussi celles qui en sont exclues, et à le justifier.

Nous entendons ainsi montrer, dans ce qui suit, qu'il n'y a pas opposition entre économie sociale et ISBL, mais au contraire que le champ des ISBL peut (et doit) constituer un chemin vers celui de l'économie sociale. 


\section{La longue marche vers un compte satellite de l'économie sociale: une étape, le compte des ISBL}

Depuis ses origines en 1982, l'Association pour le développement de la documentation en économie sociale (Addes) préconise la construction d'un compte satellite de l'économie sociale pour améliorer la connaissance de cet ensemble d'organisations, estimer la part qu'elles représentent dans l'activité économique et l'emploi et suivre d'année en année l'évolution de leur poids économique et de leur impact social.

Lors du tout premier colloque de l'Addes, en 1983, Vienney et Weber avaient délimité le champ de l'économie sociale en distinguant son noyau dur, les coopératives, les mutuelles et les associations gestionnaires, et son " halo ", les organisations paritaires, les comités d'entreprise, les fondations... Une première estimation rudimentaire de cet ensemble a été faite au cours du même colloque par Philippe Kaminski. Trois ans plus tard, en 1986, l'Insee et la DIES ayant donné leur accord pour la réalisation de ce compte satellite, un comité de pilotage a été constitué; il s'est réuni à trois reprises, sous la présidence d'André Vanoli, expert internationalement reconnu dans le domaine de la comptabilité nationale, puis a été " oublié ». Dès lors, la réalisation de ce compte satellite n'a pu être poursuivie, faute de moyens et surtout faute de volonté politique.

Sautons de 1986 à mars 2002, même si bien des choses se sont passées entre-temps. L'adoption pas l'ONU d'un manuel visant à établir un compte satellite des " institutions sans but lucratif dans le système de comptabilité nationale ", puis sa diffusion pour mise en œuvre auprès des instituts nationaux de statistique de tous les pays viennent radicalement modifier la donne et permettre au compte satellite de l'économie sociale de renaitre, tel le phénix, de ses cendres.

\section{Le manuel des institutions sans but lucratif dans le système de comptabilité nationale}

Ce manuel (Handbook of nonprofit institutions in the system of national accounts) a été rédigé par Helen Stone Tice dans le cadre d'un contrat entre le Center for civil society studies de l'université Johns Hopkins de Baltimore et la division statistique de l'Organisation des Nations unies. Après de nombreuses validations, il a été approuvé pour publication par la commission statistique de l'ONU. Publié en anglais au printemps 2003, il peut aussi être consulté en ligne sur le site: www.jhu.edu/ $\sim$ csss. Une traduction en plusieurs autres langues (dont le français) est prévue, mais non encore réalisée.

Le manuel est destiné à répondre à l'intérêt croissant pour les institutions sans but lucratif des pouvoirs publics, des citoyens, des médias et des chercheurs partout dans le monde. En effet, actuellement, les comptes nationaux sont incapables de donner une information cohérente, régulière et comparable sur ce type d'organisations, pour deux raisons. D'une part, la couverture du champ des institutions sans but lucratif reste imparfaite; 
d'autre part, les directives du SCN 93 / SEC 95 (le système international de comptabilité nationale et son application aux pays européens) conduisent à inclure de nombreuses et très importantes associations ou fondations dans les secteurs des sociétés non financières ou des administrations publiques, selon la nature de leur ressource principale (ventes ou financement public).

Ceci laisse au secteur des ISBLSM (institutions sans but lucratif au service des ménages), qui regroupe les associations et fondations vivant principalement de dons et/ou de cotisations, un champ minuscule. Or, les travaux entrepris dans le cadre des programmes Johns Hopkins de comparaison du secteur sans but lucratif dans treize puis quarante-cinq pays ont montré que les institutions sans but lucratif représentent dans certains pays européens plus de $10 \%$ de l'emploi et que ce secteur constitue donc une force économique importante et croissante.

Pour remédier à ce manque d'information qui nuit à toute politique partenariale entre l'Etat, les collectivités territoriales et les institutions sans but lucratif, le manuel demande aux instituts nationaux de statistique de:

- produire un compte satellite des institutions sans but lucratif qui regroupe l'information sur ces organisations dispersées actuellement dans les comptes nationaux entre sociétés non financières, sociétés financières, administrations publiques et institutions sans but lucratif au service des ménages;

- élargir et améliorer la couverture de ces institutions sans but lucratif par les enquêtes ou par les registres (ce qui se fait en France depuis quelques années avec l'introduction dans le répertoire Sirene de toutes les associations bénéficiant ou ayant bénéficié d'une subvention publique);

- calculer, à partir d'enquêtes auprès des ménages et des organisations, le montant (en heures de travail et en équivalent temps plein) et la valeur monétaire du travail bénévole, spécificité des institutions sans but lucratif. Le compte satellite doit évidemment rendre compte de cette contribution majeure des institutions sans but lucratif. Il apportera sur ce point un progrès décisif, cohérent avec la valorisation monétaire du travail bénévole recommandée par de nombreux ministères de tutelle aux associations quils subventionnent;

- estimer par les dépenses de fonctionnement la production non marchande des associations qui ont une activité économique principalement marchande (par exemple l'effort de formation et de qualification des travailleurs accueillis dans une entreprise d'insertion qui vend ses produits sur le marché). Cette production non marchande des associations marchandes approchera la notion encore vague de "plus-value sociale ";

- utiliser pour le compte satellite une nomenclature plus détaillée que la nomenclature d'activités française (NAF), afin de mieux distinguer les divers types d'institutions sans but lucratif;

- enfin, rassembler des indicateurs sociaux pertinents pour mesurer l'apport des institutions sans but lucratif et retracer leur rôle social, culturel et environnemental. Cette approche rejoint les propositions faites récemment par Jean Gadrey pour cerner le concept d'utilité sociale. 
En résumé, le manuel repose sur le système international de comptabilité nationale SCN 93 / SEC 95: même structure de base, mêmes concepts, mêmes sources statistiques principales. Mais il offre en plus le moyen de prendre en compte toute la palette des institutions sans but lucratif et de l'activité bénévole. Il bénéficie par ailleurs de l'expérience accumulée par les deux programmes comparatifs Johns Hopkins réalisés grâce à la coopération d'une quarantaine de pays, développés ou en développement, dont la France qui a pris part aux deux programmes.

Ce manuel a également bénéficié des avis d'un comité de pilotage, composé principalement de comptables nationaux, et du comité d'experts de l'ONU. Il a été testé par les instituts statistiques de onze pays: Australie, Belgique, Canada, Israël, Italie, Pays-Bas et Suède pour les pays industrialisés et Afrique du Sud, Argentine, Mozambique et Thaïlande pour les pays à système statistique moins développé. Tous les pays ont rempli plus ou moins complètement le cadre proposé.

Actuellement, une dizaine de pays (dont la France) ont décidé de construire un compte satellite des institutions sans but lucratif conforme au manuel. L'Italie, la Belgique et le Canada ont déjà terminé leur première campagne. Une première réunion des instituts statistiques européens s'est tenue les 11 et 12 décembre 2002 à Luxembourg, à l'initiative d'Eurostat; le manuel y a été présenté dans ses détails les plus techniques devant les représentants des instituts statistiques de vingt pays de l'Union européenne. La France en était malheureusement absente, mais elle a activement participé à une seconde réunion qui vient de se tenir (le 23 avril 2004).

\section{Où en est-on en France?}

L'Insee a pris en 2003 la décision de principe de mettre en œuvre le compte satellite des institutions sans but lucratif. La France n'est hélas plus pionnière dans cette matière, mais elle peut heureusement s'inspirer de l'exemple belge tout proche. Cette proximité n'est pas seulement linguistique, mais elle passe par la reconnaissance du concept d'économie sociale, par des formes juridiques très proches, par la similitude des problèmes économiques et sociaux rencontrés (désindustrialisation, immigration, vieillissement, insertion économique et sociale des jeunes et des chômeurs de longue durée...). Nous ne partons cependant pas de rien. Les travaux réalisés dans le cadre de l'Addes en 1997 sur les emplois et les salaires dans l'économie sociale, à partir des déclarations annuelles de données sociales (DADS), ont entretemps inspiré des initiatives régionales menées par diverses chambres régionales de l'économie sociale (Cres) et des directions régionales de l'Insee, les travaux réalisés en Midi-Pyrénées ayant permis la mise au point d'une méthodologie « clés en main » utilisable par d'autres régions.

Ceci a conduit à isoler dix objectifs proches ou immédiats.

\section{Une exploitation nationale spécifique des DADS}

Il s'agira de rééditer l'exploitation réalisée sur celle de 1995 dans le cadre de l'Addes, en tirant parti de l'expérience acquise. Cette exploitation fournira 
des données de cadrage aussi bien au compte central (ISBLSM) qu'au compte satellite. Elle commencera sur les données de l'année 2001.

\section{La constitution d'un comité de pilotage}

Dans une première phase, la fonction de celui-ci sera essentiellement d'associer à l'Insee les représentants des administrations concernées et du monde de l'économie sociale, pour les impliquer dans le projet et enrichir les réflexions.

Etablir des relations suivies avec les directions régionales de l'Insee En effet, c'est dans les DR que se gère la qualité du répertoire, c'est là que sont menées des exploitations spécifiques des DADS, que se sont nouées des coopérations avec les Cres; c'est là aussi qu’arrive et s'exprime la demande du public et des partenaires.

Rétablir le contact avec les organisations internationales

Une participation française active aux réunions internationales est un gage de crédibilité et de pérennité du projet.

Rédiger une version française résumée et pédagogique du manuel Le manuel ONU est non seulement rédigé en anglais, mais il l'est dans le sabir des comptables nationaux. Outil de référence indispensable aux professionnels, il est peu accessible aux profanes. Il s'agira donc d'en rendre la lecture accessible, voire attrayante.

\section{Jeter les bases d'un répertoire national des ISBL}

Sirene existe, des efforts continus sont faits pour améliorer sa qualité. Mais Sirene repose sur des concepts auxquels le monde associatif et l'économie sociale dans son ensemble ont du mal à s'adapter. Il faut donc créer à côté de Sirene un système de repérage spécifique, aussi richement documenté que possible, en liaison avec les travaux initiés au ministère de l'Intérieur par le bureau des associations et en veillant à ce que la classification soit compatible avec la classification ICNPO du manuel ONU.

\section{Collecter des comptes}

L'absence d'enquête annuelle d'entreprise sur un champ d'organisations par ailleurs dispensées des obligations fiscales de droit commun constitue pour le statisticien un handicap rédhibitoire. Or, il faut connaitre les comptes d'une ISBL pour savoir si elle fait partie des administrations publiques, des sociétés non financières ou des ISBLSM. Et il faut que ces comptes soient convenablement normalisés. Si la Belgique a pu, si rapidement, se construire un compte des ISBL, c'est qu'elle peut s'appuyer sur une enquête spécifique, ce qui nous fait cruellement défaut. Des opérations ponctuelles de collecte de comptes en France, destinées à alimenter la construction du compte satellite, devront être beaucoup plus directives que celles qui les ont précédées. 


\section{Actualiser l'information existante sur les ménages} (enquête Insee conditions de vie)

Les enquêtes auprès des ménages ou des personnes, concernant leurs dons aux œuvres, le temps consacré au bénévolat, leurs relations entretenues avec les ISBL et leurs opinions sur celles-ci fournissent des informations complémentaires à celles que l'on peut collecter auprès des organismes ou à travers les sources administratives.

\section{Procéder à une évaluation systématique des sources existantes}

On sait qu'il existe, dans les fédérations, dans les ministères, dans la comptabilité des collectivités territoriales, quantité de sources administratives et comptables portant sur telle ou telle partie du champ ISBL. Quatre directions d'investigations semblent prioritaires: le répertoire Finess; la fiscalité; le suivi de la dépense publique; l'immatriculation des associations au ministère de l'Intérieur.

\section{Mettre en place des procédures de travail légères, réactives et dynamiques}

Autour d'un groupe de travail restreint et disposant de peu de moyens, restrictions budgétaires obligent, il faudra utiliser au maximum les échanges électroniques et envisager à terme le développement d'un site Web dédié au projet.

Ce rapide historique étant retracé et les projets d'avenir étant balisés, il est important de comparer la manière dont les institutions sans but lucratif, qui sont une partie de l'économie sociale, sont définies dans le système de comptabilité nationale lui-même, par rapport aux précisions complémentaires que donne le Manuel. D'autre part, il faut avoir une idée claire de la distinction entre les ISBL (du compte satellite) et les ISBLSM (institutions sans but lucratif au service des ménages), les seules à être lisibles dans les comptes nationaux annuels. 